\title{
Investigation of grain boundary and orientation effects in polycrystalline metals by a dislocation-based crystal plasticity model
}

\author{
Jianqiao $\mathrm{Hu}^{\mathrm{a}}$, Zhuo Zhuang ${ }^{\mathrm{b}}$, Fengxian Liu ${ }^{\mathrm{b}}$, Xiaoming Liu ${ }^{\mathrm{a}, *}$, Zhanli Liu ${ }^{\mathrm{b}, *}$ \\ ${ }^{a}$ State Key Laboratory of Nonlinear Mechanics, Institute of Mechanics, Chinese Academy of Sciences, Beijing 100190, PR China \\ ${ }^{\mathrm{b}}$ Applied Mechanics Laboratory, School of Aerospace Engineering, Tsinghua University, Beijing 100084, PR China
}

\section{A R T I C L E I N F O}

\section{Keywords:}

Dislocation-based crystal plasticity

Polycrystalline metal

Grain size effect

Grain orientation effect

\begin{abstract}
A B S T R A C T
Polycrystalline metal with well-designed grain structure exhibits different strength and ductility compared with the conventional macro scale materials. In the present study, the mechanisms of strain hardening and inhomogeneous deformation in polycrystals are analyzed by considering the interaction between dislocations and grain boundaries. Firstly, a dislocation density based crystal plasticity model is developed and then used to quantitatively study the grain size and orientation effects in polycrystals. With the decrease of grain size, the accumulation and interaction of dislocations are promoted in the grain, resulting in back-stress hardening. Simultaneously, two opposite effects of the inhomogeneity in grain orientation are obtained by evaluating the local Schmidt factors in each grain and their standard deviation. The findings highlight the effect of grain boundary on the strength and provide quantitative insight into the effect of grain orientation on ductility.
\end{abstract}

\section{Introduction}

In recent decades, the nanocrystalline (NC) materials, with average grain size typically at the level of about or less than $100 \mathrm{~nm}$, have attracted widespread attention due to their superior mechanical strength compared to the conventional coarse-grained (CG) metals. However, for most NC metals, their ductility under tension is not satisfactory. The poor ductility of these NC materials has become a seemingly insurmountable obstacle for widespread applications. Several published literatures have revealed the reasons for the low ductility of these NC materials [1,2], a widely accepted interpretation is that small grains tend to induce stress concentration, where the nucleation and propagation of micro-cracks would start. Partly because of these reasons, the structural applications of bulk nanostructured materials shifted towards ultrafine-grained metals, for which the grain sizes are above $\sim 100 \mathrm{~nm}$ but below $1 \mu \mathrm{m}$. Some polycrystalline metals with well-designed nanostructures have been reported, which can achieve a balanced combination of high strength and excellent ductility [3,4]. The basic strategy to improve tensile ductility of metals without loss of strength is elaborately building a specific distribution of crystalline grains with different sizes by a thermo-mechanical treatment [5] or the gradient microstructures [6]. It indicates that the grain characteristics of processed materials have a strong influence on the mechanical properties. Therefore, it is of significance to clarify the role of different grains for material design.

To further investigate the deformation mechanism of polycrystalline metals, especially the effect of grain size, crystal plasticity models $[7,8]$ have been developed and the effect of grain size on hardening is assumed by the empirical Hall-Petch relationship $[9,10]$. That is, the yield stress is proportional to the inverse square root of the mean grain size, which is suitable for the polycrystals with micrometer-sized grains. By taking into account the Hall-Petch relationship at the grain level, a finite element model [11] has been revisited for predicting the mechanical behaviors of ultrafine-grained Nickels. In the work of Lin et al. [12], a finite element based model was developed with quantized crystal plasticity to study distinctive features of NC metal. The quantized slip events suggested that the magnitude of the largest strain burst was inversely proportional to grain size. These prior efforts have predicted the effects of grain size on mechanical response, both flow rule and hardening law are mostly phenomenological and based on the conventional Hall-Petch relationship. However, these models are not able to capture the evolution of internal microstructures, e.g. dislocations.

Due to the limitations of phenomenological models, a number of crystal plasticity models based on dislocation density have been developed $[13,14]$ since the plastic flow of metals is mainly controlled by dislocation motion. Arsenlis et al. [15] developed a dislocation density based model by incorporating the combined evolution of statistically

\footnotetext{
* Corresponding authors.

E-mail addresses: xiaomingliu@lnm.imech.ac.cn (X. Liu), liuzhanli@tsinghua.edu.cn (Z. Liu).
} 
stored and geometrically necessary densities (SSDs and GNDs), and investigated the effect of grain boundaries on the stress-strain behavior and the stress concentrations for the constrained polycrystalline thin films. In addition to the conventional analysis based on SSDs and GNDs, another effective strategy to establish the crystal plasticity model is to gain insight into the simulation results from discrete dislocation dynamics (DDD). The discrete dislocation plasticity theories, focus on the dynamics of individual dislocations, have obtained interesting results about the behavior of dislocations [16-21]. In particular, the Bauschinger effect in the films with multiple grains across the thickness was investigated by a two-dimensional DDD simulation [22]. The strengthening mechanism in polycrystals with penetrable and impenetrable grain boundaries has been studied [23] and the simulation results indicate that the Hall-Petch relation can be achieved for both cases. The DDD simulation approach was also extended to account for grain boundary sliding and the absorption, emission and transmission of lattice dislocations at grain boundaries [24]. Nevertheless, it is still of great challenge to develop a dislocation-based plasticity law from DDD results due to the discrete distributions and dynamics of dislocations [25]. In addition, most DDD simulations only consider the case of free surface and dislocations can easily escape from the surface, whereas dislocation can be trapped by the grain boundaries in polycrystalline metals. By carrying out DDD simulations, Cui et al. [26] have investigated the confined plasticity in coated pillars at submicron scale. The simulation results have clearly indicated that the dislocations were trapped by the surface. The back stress is generated in this way that causes the strain hardening in coated pillars. Since dislocations can pile up on the grain boundaries, similar hardening mechanism also underlays the deformation in polycrystalline metals. The inspiration to develop a dislocation-based plasticity model stems from the similarity of the coating to grain boundaries.

In this study, a dislocation-based crystal plasticity (DCP) model is developed from the simulation results of the previous three-dimensional DDD [26] and described in detail in Section 2. The DCP model is then verified in Section 3. A series of numerical simulations are carried out in Section 4 to investigate the effects of grain boundary and grain orientation on the compressive plasticity in polycrystalline nickel. The relationship between mechanical behavior and corresponding dislocation distributions are studied. Finally, some discussions and conclusions are summarized.

\section{Dislocation-based crystal plasticity model}

In this section, the DCP model is formulated. Firstly, we emphasize the inheritance from our previously DDD simulations [26] and summarize the constitutive law of an individual grain. This hierarchical multi-scale calculation provides a physical basis to investigate the dislocation-based plasticity of materials. Then, the elastoplastic crystal model is introduced in the finite element framework to study the plastic behavior at the grain scale.

\subsection{Constitutive law from discrete dislocation dynamics}

By carrying out three-dimensional DDD simulations [26], we have achieved an insight into the microscopic deformation mechanisms as well as the dislocation evolutions in confined plasticity. Dislocations have similar behaviors in the crystalline grain and the confined single crystalline specimen. The internal dislocations produce plasticity, and dislocations trapped by the grain boundaries or coated surface give rise to back stress. It is also necessary to illuminate that only single arm dislocation sources (SAS) are considered in the simulations since they are the dominant dislocation mechanism at submicron scale $[27,28]$. The grain size that we focus on in this work is also in the range of submicron scales where the SAS mechanism is dominant.

As plastic deformation in metal materials is caused by the motion of dislocations, a continuum description of collective dislocations is important in crystal plasticity. Starting from the equation of dislocations motion in DDD simulations and similar to previous work $[29,30]$, the motion of collective dislocations of slip plane $\alpha$ can be derived as

$B \mathbf{v}^{(\alpha)}=b \cdot\left(\tau^{(\alpha)}+\tau_{b}^{(\alpha)}+\tau_{o}^{(\alpha)}\right)$

where $B$ is drag coefficient, $\mathbf{v}^{(\alpha)}$ is the average dislocation velocity vector along the slip direction $\mathbf{s}^{(\alpha)}, \mathbf{v}^{(\alpha)}=v^{(\alpha)} \mathbf{s}^{(\alpha)} . b$ is the magnitude of Burgers vector, $\tau^{(\alpha)}$ is the external driving force vector, $\tau_{b}^{(\alpha)}$ is the back stress vector and $\tau_{0}^{(\alpha)}$ is the slip resistance vector including lattice friction and the interaction between dislocations. The three force vectors above are defined as

$\tau^{(\alpha)}=\tau^{(\alpha)} \mathbf{s}^{(\alpha)}=\left(\mathbf{s}^{(\alpha)} \cdot \sigma \cdot \mathbf{m}^{(\alpha)}\right) \mathbf{s}^{(\alpha)}$

$\tau_{b}^{(\alpha)}=-\tau_{\text {back }} \mathbf{s}^{(\alpha)}$

$\tau_{o}^{(\alpha)}=-\operatorname{sgn}\left(\tau^{(\alpha)}-\tau_{\text {back }}\right) \tau_{o p e n} \mathbf{s}^{(\alpha)}$

Here, $\mathbf{s}^{(\alpha)}$ and $\mathbf{m}^{(\alpha)}$ represent the slip direction and slip plane normal in the slip system $\alpha$, respectively. $\sigma$ is the stress tensor. $\tau_{\text {back }}$ is the back stress due to dislocations trapped at the grain boundaries. $\tau_{\text {open }}$ is the stress to activate the internal dislocation source. 'sgn' is the sign function. In the study, all grain boundaries are assumed to be impenetrable to dislocations $[22,31]$, the back stress $\tau_{\text {back }}$ and the activation stress $\tau_{\text {open }}$ are delivered from the DDD simulations of the confined plasticity [26] as follows

$\tau_{\text {open }}=\tau_{0}+\alpha_{0} \mu b \sqrt{\rho_{m}}+\frac{k_{s} \mu}{\lambda / b}$

$\tau_{\text {back }}=\alpha_{0}^{\prime} \rho_{t} b \mu d$

where $\tau_{0}$ is lattice friction stress, $\mu$ is shear modulus, $\rho_{m}$ and $\rho_{t}$ are the total mobile and trapped dislocation densities of all slip systems in a grain, respectively, $\alpha_{0}$, and $\alpha_{0}^{\prime}$ are all dimensionless constants, $\lambda$ is effective SAS length and related to the grain size, and $d$ is the equivalent diameter of grain. It is worth pointing out a concept of the sign of trapped dislocation density $\rho_{t}^{(\alpha)}$ is introduced to characterize the directions of force vectors $\tau_{b}^{(\alpha)}$ and $\tau_{o}^{(\alpha)} . \rho_{t}^{(\alpha)}$ is positive when it is accumulated in the same direction as $\mathbf{s}^{(\alpha)}$, otherwise, it is negative. It also should be noted that Eq. (1) is only valid when

$\left|\tau^{(\alpha)}+\tau_{b}^{(\alpha)}\right|>\left|\tau_{0}^{(\alpha)}\right|$

Otherwise, $v^{(\alpha)}=0$. When the average dislocation velocity $v^{(\alpha)}$ is obtained, the plastic shear strain rate on the slip plane can be evaluated via Orowan's equation

$\dot{\gamma}_{p}^{(\alpha)}=\rho_{m}^{(\alpha)} b v^{(\alpha)}$

For small deformation problems, the plastic velocity gradient tensor $\mathbf{L}_{P}$ can be written as

$\mathbf{L}_{p}=\sum_{\alpha} \dot{\gamma}_{p}^{(\alpha)} \mathbf{s}^{(\alpha)} \otimes \mathbf{m}^{(\alpha)}$

Once the operation of dislocations occurs, the evolutions of both mobile and trapped dislocation densities are

$$
\begin{gathered}
\dot{\rho}_{t}^{(\alpha)}=\frac{2(1+\cos \beta)}{b d} \cdot \dot{\gamma}_{p}^{(\alpha)} \\
\dot{\rho}_{m}^{(\alpha)}=\left[\frac{1}{2 b \lambda}-\frac{2(1+\cos \beta)}{b d}\right] \cdot \dot{\gamma}_{p}^{(\alpha)}
\end{gathered}
$$

where $\beta$ is the angle between the normal direction of slip plane $\alpha$ and the axial direction of the sample.

To further illustrate the three force vectors in Eq. (1), a schematic diagram for the direction of the force vectors under cyclic loading, with the assumption that the mobile dislocation density is constant, is shown in Fig. 1. The symbol ' +' means the force vector is in the same direction as the slip direction $\mathbf{s}^{(\alpha)}$ while the ' - ' indicates the opposite. 


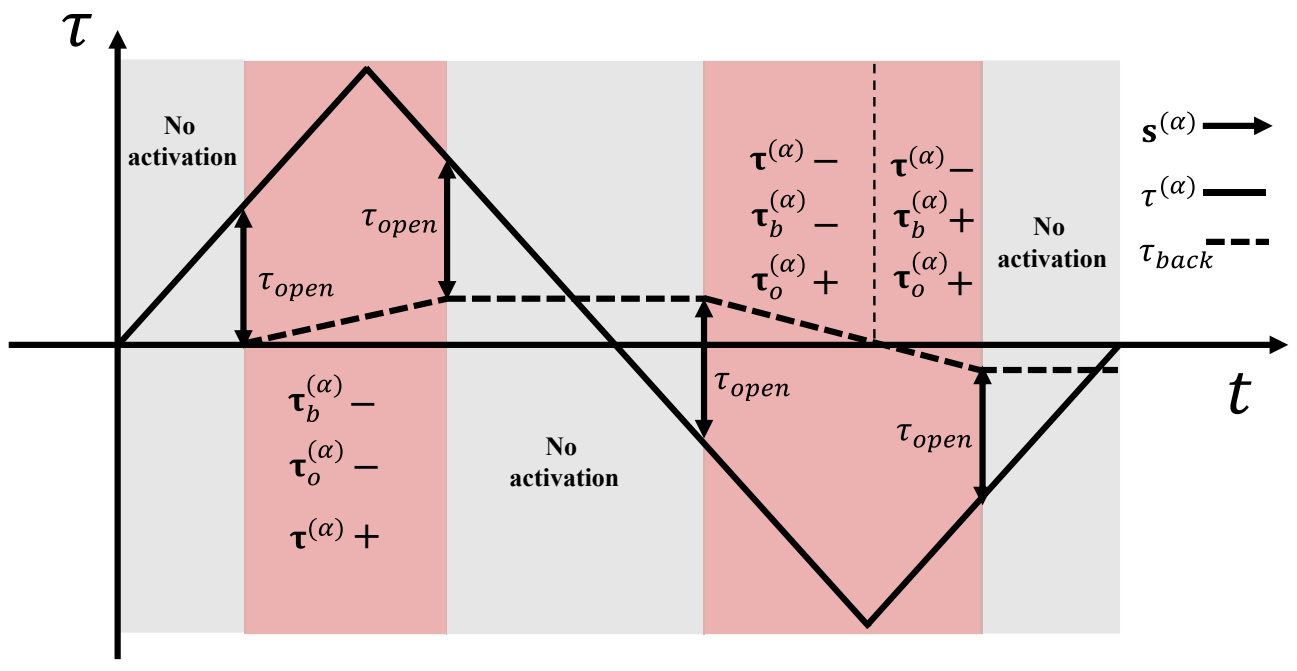

Fig. 1. Direction instruction of force vectors during a cyclic load.

\subsection{Finite element analysis at the grain scale}

The DCP model is performed in the framework of finite element analysis at the grain scale. To simulate the mechanical behavior of crystalline grain more accurately, each grain contains numerous elements in the finite element analysis as shown in Fig. 2. Thus, two characteristic lengths are included in the finite element analysis at the grain scale. One is the grain size $d$ and the other is the element size $l$. The Burgers vector $\mathbf{b}$ and slip plane $\mathbf{n}$ are defined in the grain, i.e. all elements in one grain share the same grain orientation. In this study, the slip direction is taken as the same direction as the Burgers vector. The mobile and trapped dislocation densities are calculated in each element to describe their inhomogeneous distribution in the crystalline grain, the initial dislocation densities of all elements in the same grain are set to the same and the subsequent evolution of dislocation densities is dependent on the grain size as Eq. (10).

The calculation mainly contains the following procedures: (i) The stress state, dislocation densities and grain orientation at current step are obtained to confirm whether the dislocation activation occurs. (ii) If the criteria Eq. (7) is met, the motion of collective dislocations is calculated to evaluate the plastic velocity gradient tensor. The mobile and trapped dislocation densities are then evolved according to Eq. (10). Next, the plastic deformation rate $\dot{\varepsilon}^{p}$ and the plastic spin rate $\dot{\omega}^{p}$ can be obtained by:

$\dot{\boldsymbol{\varepsilon}}^{p}=\frac{1}{2} \cdot\left(\mathbf{L}^{p}+\mathbf{L}^{p \mathrm{~T}}\right)$

$\dot{\omega}^{p}=\frac{1}{2} \cdot\left(\mathbf{L}^{p}-\mathbf{L}^{\mathrm{pT}}\right)$

(iii) Afterwards, we get the objective rate of stress $\dot{\sigma}$ :

$\dot{\sigma}=\mathbf{C}^{e}:\left(\dot{\boldsymbol{\varepsilon}}-\dot{\boldsymbol{\varepsilon}}^{\mathrm{p}}\right)$

where $\mathbf{C}^{e}$ is tensor of elastic modulus, $\dot{\varepsilon}$ is deformation rate tensor. The

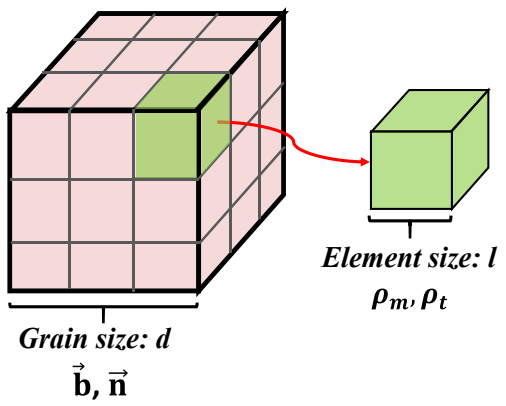

Fig. 2. Schematic for finite element analysis at the grain scale. equilibrium stress field associated with the homogenized plastic strain is calculated by finite element analysis in a unified continuum mechanics framework, which has the following general forms [32-34]:

$\mathbf{M u}+\mathbf{F}^{\text {int }}=\mathbf{F}^{\text {ext }}$

$\mathbf{M}=\int_{\Omega} \rho \mathbf{N}^{\mathrm{T}} \mathbf{N} d \Omega$

$\mathbf{F}^{i n t}=\int_{\Omega} \mathbf{B}^{\mathrm{T}} \sigma d \Omega$

$\mathbf{F}^{e x t}=\int_{\Gamma} \mathbf{B}^{\mathrm{T}} \mathrm{T}^{0} d \Gamma+\int_{\Omega} \mathbf{B}^{\mathrm{T}} \sigma^{0} d \Omega$

$$
\mathbf{u}=\mathbf{u}^{0} \quad \Gamma \in \Gamma_{\mathrm{u}}
$$

$\mathbf{T}=\mathbf{T}^{0} \Gamma \in \Gamma_{\mathrm{f}}, \quad \mathbf{T}=0 \Gamma \notin\left(\Gamma_{\mathrm{u}} \cup \Gamma_{\mathrm{f}}\right)$

where $\mathbf{M}$ is mass matrix, $\mathbf{N}$ is the shape function vector and $\mathbf{B}=\operatorname{grad}[\mathbf{N}] . \mathbf{F}^{\text {int }}$ is the internal force induced by the total stress $\boldsymbol{\sigma} . \mathbf{F}^{\text {ext }}$ is the external force resulting from two parts, one is the applied traction $\mathbf{T}^{0}$ and the other is the initial stress field $\sigma^{0}$, which is introduced to represent the preexisting defects in crystal.

The characteristic of finite element analysis in the study is that each element belongs to a certain grain and own its self-governed constitutive law which is dependent on the grain size and orientation. The advantages of this treatment are that the force balance and displacement continuity between adjacent crystalline grains can be achieved spontaneously under the conventional framework of finite element analysis. More importantly, the DCP model has a brief form and a small number of parameters can be obtained by fitting the experimental data or from the numerical simulations of smaller scale, such as DDD or molecular dynamics.

\section{Convergence and parameter determination}

In the present work, the DCP model is carried out to investigate the stress-strain relation of nickel and compare with the DDD results. The material properties of nickel are as follows: shear modulus $\mu$ is $76 \mathrm{GPa}$, Poisson's ratio $\nu$ is 0.31 , the magnitude of Burgers vector $b$ is $0.26 \mathrm{~nm}$ and the friction stress $\tau_{0}$ is taken as $11 \mathrm{MPa}$ for nickel [35]. Two issues are concerned in this section. Firstly, as illustrated in Fig. 2, numerous elements are contained in one crystalline grain, the issue emerges that how many elements in one grain can obtain an accurate mechanical response. Secondly, the parameter selection is discussed and clarified. In the DCP model, the dimensionless constants $\alpha_{0},, \alpha_{0}^{\prime}$ as well as the drag coefficient $B$ require carefully determined, in which, $\alpha_{0}$ is usually taken as 0.5 and is set at 1.0 to characterize the interaction between 
(a)

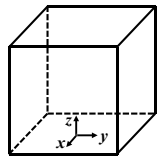

Cube: $0.8 \times 0.8 \times 0.8 \mu \mathrm{m}^{3}$

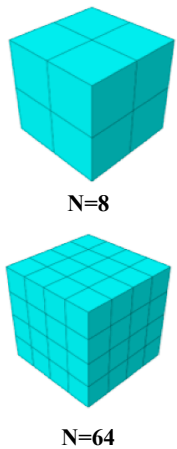

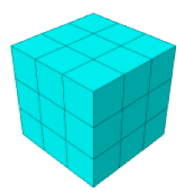

$\mathbf{N}=\mathbf{2 7}$

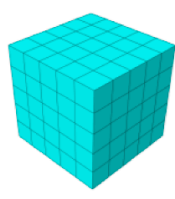

$\mathbf{N}=\mathbf{1 2 5}$ (b)

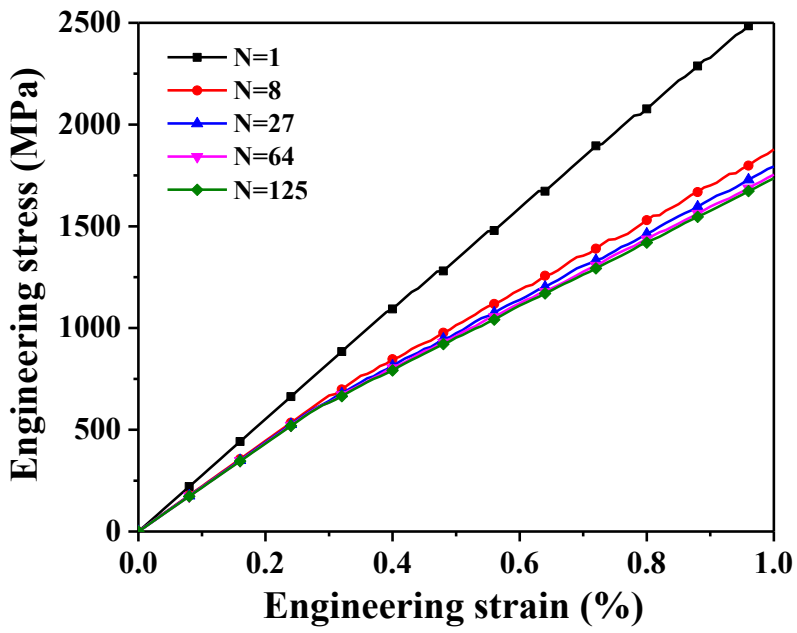

Fig. 3. (a) $\mathrm{N}$ elements in one grain, (b) strain-stress curves for a grain with $\mathrm{N}$ elements.

dislocations and the line tension stress to activate the SASs [35,36], respectively. Since the rate effect is not considered in this work, similar to the literature [30], a dimensionless parameter $B_{N}=B \dot{\varepsilon} / \tau_{0}$ is proposed to discuss the influence of viscosity.

\subsection{Convergence test of elements}

A uniaxial compression of nickel cube with side length $0.8 \mu \mathrm{m}$ is studied with element number $\mathrm{N}=1,8,27,64$ and 125 , as shown in Fig. 3(a). The initial dislocation density of crystalline grain is $100 \mu \mathrm{m}^{-2}$ and there is no trapped dislocation at the beginning. The normal vector of slip plane $\mathbf{n}$ and the Burgers vector $\mathbf{b}$ are defined as one of $\{111\}$ and $\langle 110\rangle$ separately under the condition that the two vectors are orthogonal, which are the typical FCC slip systems. In the simulation, $\alpha_{0}^{\prime}$ is temporarily set to 0.2 and $B_{N}$ is $9 \times 10^{-7}$.

A uniaxial compression is performed under displacement controlled mode along [ [ 001 1] crystal orientation. The stress-strain responses for a grain with different number of elements are shown in Fig. 3(b). It can be seen that one element (i.e. $\mathrm{N}=1$ ) cannot accurately describe the deformation behavior of the grain. While for the other cases, after the initial elastic deformation, the stress reaches the yield point. Then the hardening process happens subsequently, it indicates that the trapped dislocation density is constantly accumulating as loading continues. In the following simulations, at least 27 elements are contained in a grain.

\subsection{Parameter determination}

As mentioned previously, a few parameters in the DCP model need to be determined. Except $\alpha_{0}$ and in Eq. (5), the dimensionless viscosity parameter $B_{N}$ and back stress related parameter $\alpha_{0}^{\prime}$ are discussed in this section. In particular, Eq. (1) describes the motion of dislocations in a continuum manner via a linear viscosity and preserves some nature of dislocation dynamics. To model the rate-independent mechanical behavior in crystals, an appropriate value of drag coefficient $B$ should be used. In the DDD simulation [26], the diameters of nickel micropillars are varied from 0.2 to $0.8 \mu \mathrm{m}$ and the ratio of height to diameter is fixed to 2 . As a comparison, pillars with diameter of $0.4 \mu \mathrm{m}$ and $0.8 \mu \mathrm{m}$ are also simulated by the DCP model to clarify the concerned parameters.

Firstly, different values of $B_{N}$ are selected to discuss the rate-independent mechanical behavior and $\alpha_{0}^{\prime}$ is set to 0.2 , a uniaxial compressive load is performed on the top of $0.4 \mu \mathrm{m}$ diameter pillar along [ $\left.\begin{array}{lll}0 & 0 & 1\end{array}\right]$ crystal orientation. The effect of normalized drag coefficient $B_{N}$ on the stress-strain relation is shown in Fig. 4. It can be seen that there is nearly no difference between the curves when the normalized drag

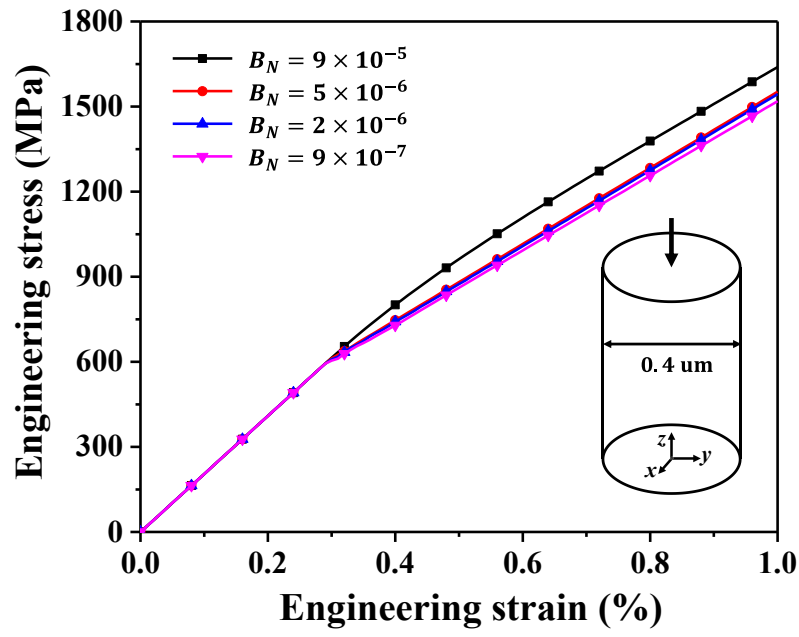

Fig. 4. Strain-stress curves of nickel pillar at different normalized drag coefficient $B_{N}$.

coefficient is $\sim 10^{-6}$ or smaller. Therefore, $B_{N}$ is set to $2 \times 10^{-6}$ in the following simulation.

The dimensionless constant $\alpha_{0}^{\prime}$ determines the back stress, which affects the hardening process. The value of $\alpha_{0}^{\prime}$ can be obtained by fitting the DDD results. The simulation result at different values of $\alpha_{0}^{\prime}$ is shown in Fig. 5(a), and $\alpha_{0}^{\prime}$ is determined to be as 0.08 since the result obtained by the DCP model at this value matches well with the DDD model. To further verify the validity of this value, nickel pillar with diameter of $0.8 \mu \mathrm{m}$ is also simulated as shown in Fig. 5(b). Due to the nature of discrete dislocations in DDD model, the stress-strain curves are much smoother in the DCP model. Despite this, the two sets of curves are generally in good agreement and the value of $\alpha_{0}^{\prime}$ is set to 0.08 in the following simulations.

As indicated above, all the parameters in the DCP model have been determined for nickel and the DCP model has been clarified to simulate the mechanical behavior of crystalline grains effectively.

\section{Simulation setup and results}

In this section, the plastic behavior of polycrystalline nickel is investigated by using the DCP model. The nickel sample contains a welldesigned distribution of grains. To minimize the effect of sample size itself, the image force caused by free surface is not considered in the 
(a)

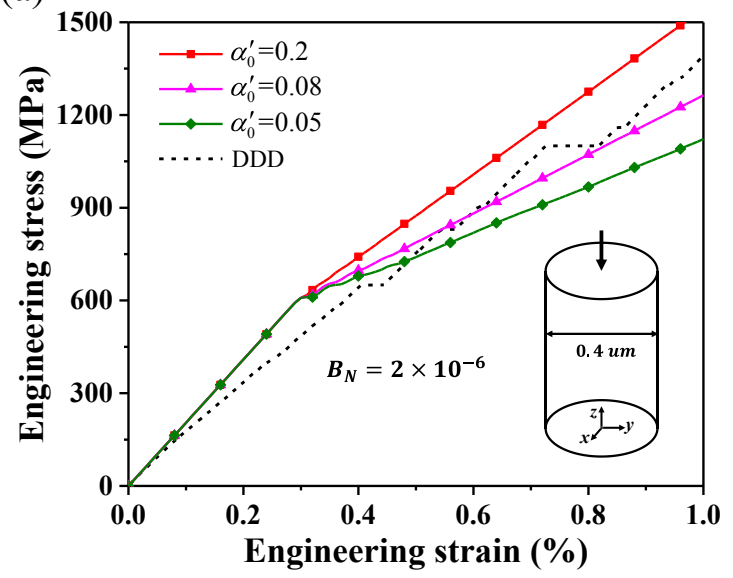

(b)

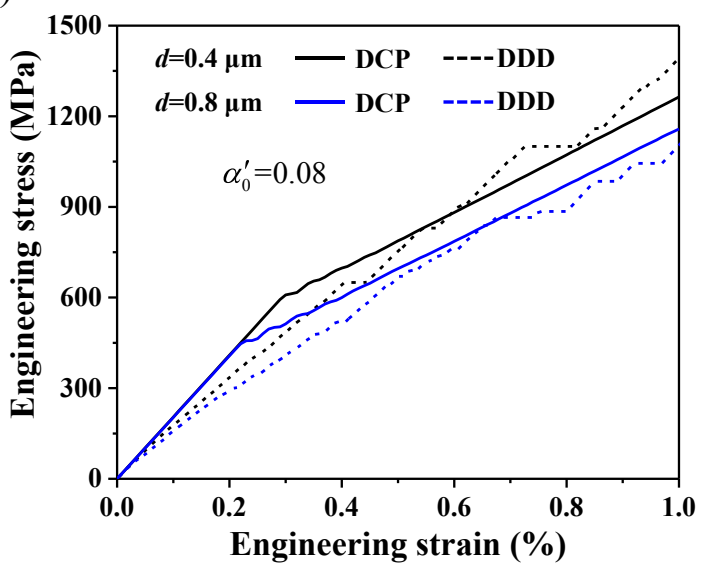

Fig. 5. (a) Strain-stress curves at different $\alpha_{0}^{\prime}$, (b) comparison of the stress-strain curves between DCP and DDD models.

simulation. The cross section of all samples is square with side length $4.8 \mu \mathrm{m}$, with the ratio of height to side length fixed to 2 . The effects of grain boundary and orientation are studied in this section. The grain boundary is simulated by setting different grain sizes ranging from 0.4 to $1.2 \mu \mathrm{m}$ in the samples. For each crystalline grain, the initial dislocation density is randomly defined in the range of $40-60 \mu \mathrm{m}^{-2}$ and no trapped dislocations are introduced at the beginning. To investigate the effect of grain orientation, the normal vector of slip plane $\mathbf{n}$ and Burgers vector $\mathbf{b}$ of each grain in the simulation are defined as the following two ways: (i) $\mathbf{n}$ and $\mathbf{b}$ are one of $\left\{\begin{array}{lll}1 & 1 & 1\end{array}\right\}$ and $\left\langle\begin{array}{lll}1 & 1 & 0\rangle\end{array}\right.$ separately under the condition that the two vectors are orthogonal, (ii) $\mathbf{n}$ and $\mathbf{b}$ are randomly defined in the orthogonal case to model the disordered grain orientation. The former way achieves an ordered grain orientation similar to the twelve FCC slip systems while the latter one obtains a disorganized configuration.

\subsection{Effect of grain boundary}

The effect of grain boundaries is investigated by designing different grain sizes in nickel. In the calculation, the grains are set to be cube and their side lengths are $0.4,0.6,0.8,1.2 \mu \mathrm{m}$, respectively. Firstly, $\mathbf{n}$ and $\mathbf{b}$ are defined as the former way above to achieve the ordered grain orientation. The initial dislocation densities for all grains are in the range of $40-60 \mu \mathrm{m}^{-2}$ as mentioned previously. As shown in Fig. 6(a), the engineering stress-strain response of nickel samples with different grain sizes as well as the schematic diagram for the sample with grain size of
$1.2 \mu \mathrm{m}$ is illustrated. The well-known size effect can be observed, i.e. the sample with larger grain size exhibits a lower yield stress. The yield stresses at strain offsets of $0.0 \%$ and $0.2 \%$ are also marked in Fig. 6 (a). Soon after the initial yielding, the strain hardening process starts. Particularly, for sample with grain size of $0.8 \mu \mathrm{m}$, when the strain reaches about $0.2 \%$, the sample starts to yield, i.e., deviating from the linear elastic stress-strain response, at about $401 \mathrm{MPa}$, which is consistent with the experimental data [37].

To further illustrate the effect of grain boundaries, the yield stresses initially deviated from elasticity $\left(0.0 \%\right.$ offset) and when $\varepsilon_{p}=0.2 \%$ $(0.2 \%$ offset $)$ are extracted, as shown in Fig. 6(b), respectively. The boundary space is considered to be the same as the grain size here. By fitting these two sets of yield stresses, we can obtain the relationships that $\sigma_{0.0}=375 \times d^{-0.38}$ and $\sigma_{0.2}=601 \times d^{-0.66}$, where $\sigma$ is in the unit of MPa and $d$ is $\mu \mathrm{m}$. The initial yield strength at $0.0 \%$ offset is only related to the grain size, since no dislocations are trapped at this time and there is no back stress. While the increase in yield stress at the case of $0.2 \%$ offset is attributed to the pile-ups of dislocations at the grain boundary. It can be seen that the grain boundary greatly strengthens the back stress hardening, the size-dependent exponent increases from 0.38 to 0.66 , which is consistent with the experimental data quantitatively [38]. Interestingly, despite the strain hardening phenomenon in specimen with smaller grains is more pronounced under the same plastic deformation, the stress levels in different samples exhibit a unified dimensional dependence. It motivates us to explore the inherent deformation mechanism. The involved length scales in this issue is the (a)

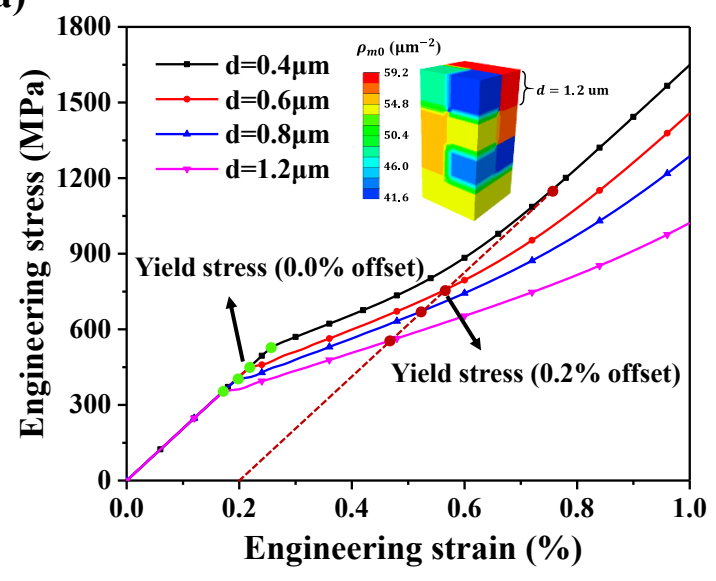

(b)

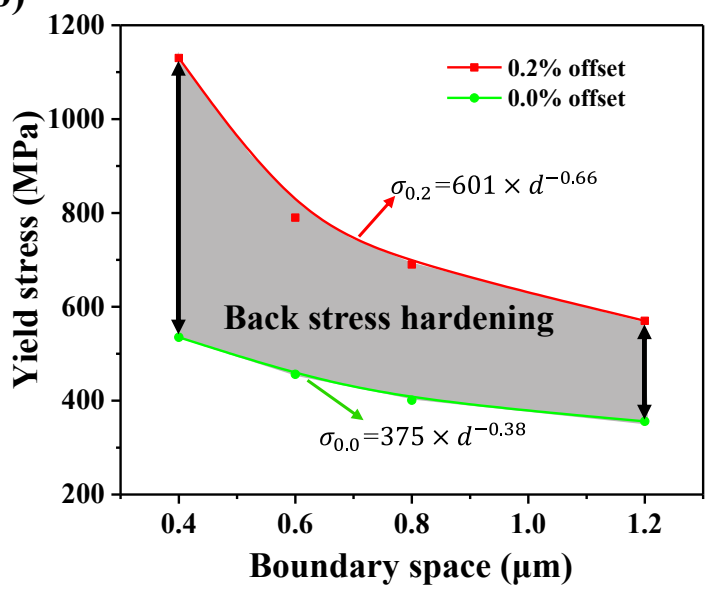

Fig. 6. (a) Strain-stress curves for polycrystals with different grain sizes, (b) the yield stress at different strain offsets vs. the grain boundary space. 
(a)

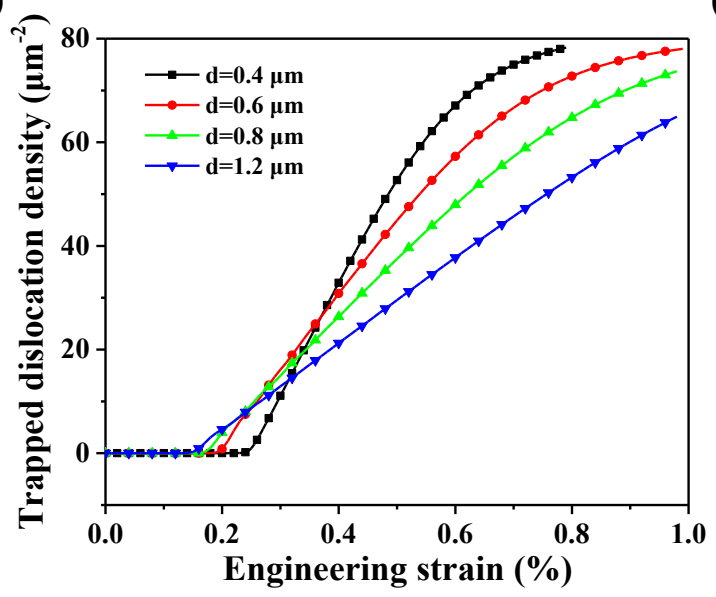

(b)

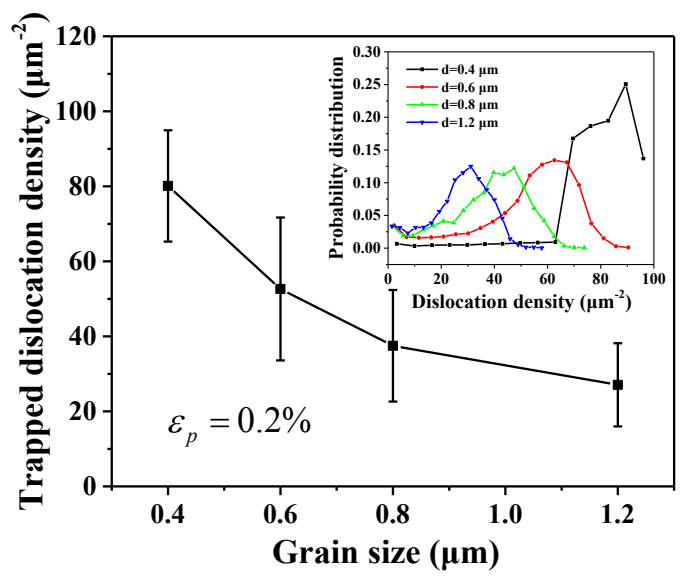

(c)

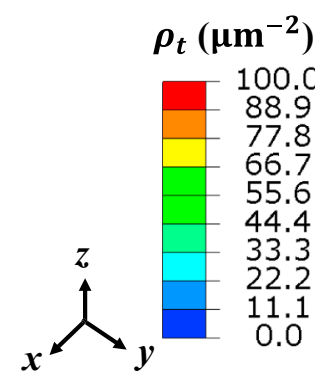

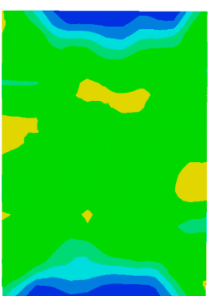

$\mathrm{d}=\mathbf{0 . 6 \mu \mathrm { m }}$

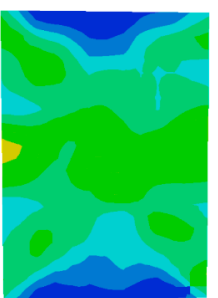

$\mathrm{d}=0.8 \mu \mathrm{m}$

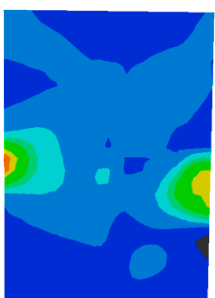

$\mathrm{d}=1.2 \mu \mathrm{m}$

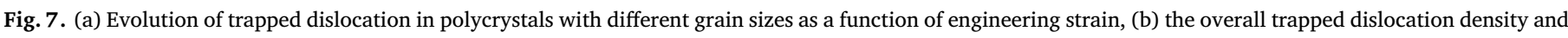

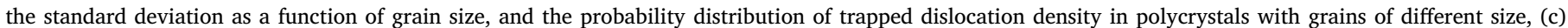
distribution of trapped dislocation in polycrystals with different grain sizes when $\varepsilon_{p}=0.2 \%$.

obstacle spacing in terms of forest dislocations and the grain size. With the decrease in the boundary space (i.e. the grain size), the mobile dislocations tend to be trapped by the grain boundaries, which leads to more pronounced back stress. In this case, the uniform size dependence law would be still expected when the external load continues. Moreover, since the situation in which the dislocations penetrate the grain boundary and the mutual annihilation of dislocations at grain boundaries are also possible and not considered in the model, the numerical results maximize the effect of trapped dislocation-induced back stress, the size-dependent exponents obtained by most of the experimental data would fall in the range between 0.38 and 0.66 [39,40], as the gray area shown in Fig. 6(b).

To further investigate the back stress, as shown in Fig. 7(a), the evolution of overall trapped dislocation density is calculated by the volume weight average of dislocation density within each grain in a sample. It can be seen that the trapped dislocation density increases more rapidly in polycrystals with small grains at the initial stage. It is consistent with the cognition that the internal dislocations are more likely to be trapped by the grain boundaries in the case of small boundary space. Subsequently, as the dislocations gradually accumulate on the grain boundaries, the back stress also increases. It makes the mobile dislocations more difficult to be activated, so that the increase in trapped dislocation density becomes slow. In addition, the overall trapped dislocation density and the standard deviation of dislocation densities in all grains of a sample are evaluated, as shown in Fig. 7(b). Besides, the probability distribution of trapped dislocation densities is also presented in Fig. 7(b). It can be seen that the overall trapped dislocation density is higher in sample with small grains, which can also be clarified by its probability distribution. As the grain size increases, the trend of decreasing the dislocation density gradually slows down. This may also be the reason why the strengthening effect of back stress is weakened when the grain size becomes larger. Furthermore, the distribution of trapped dislocations in samples with different grain sizes are also presented in Fig. 7 (c). When the plastic strain is $0.2 \%$, the dislocation distribution in sample with small grains $(d=0.4 \mu \mathrm{m})$ becomes non-uniform and the local trapped dislocation density can be as high as $99.4 \mu \mathrm{m}^{-2}$. This heterogeneous distribution of dislocations tends to cause the stress concentrations, leading to the nucleation of micro-cracks or other defects. This result revisits the traditional contradiction between high strength and ductility. Due to a large number of internal grain boundaries, the deformation incompatibility arises and this leads to poor ductility.

\subsection{Effect of grain orientation}

To clarify the effect of grain orientation, polycrystalline nickel with a grain size of $1.2 \mu \mathrm{m}$ are simulated in this section. For each grain in the polycrystals, the normal vector of slip plane $\mathbf{n}$ and Burgers vector $\mathbf{b}$ are defined by the two ways mentioned above, corresponding to the case of ordered and disordered grain orientations, respectively. Since the mobile dislocations on the slip system with largest Schmidt factor are most easily activated and dominate the plastic deformation, the Schmidt factor for this slip system is used to characterize the local grain orientation. The Schmidt factor $\mathrm{M}_{i}$ of each grain $i$ (appointed as the local Schmidt factor) in the polycrystals is calculated by:

$\mathrm{M}_{\mathrm{i}}=\left|\frac{\mathbf{n} \cdot \mathbf{L}}{|\mathbf{n}| \cdot|\mathbf{L}|}\right| \cdot\left|\frac{\mathbf{b} \cdot \mathbf{L}}{|\mathbf{b}| \cdot|\mathbf{L}|}\right|$

where $\mathbf{L}$ is the unit vector of load direction. After obtaining the Schmidt factor of each grain in the polycrystals, the overall Schmidt factor $\bar{M}$ is evaluated by averaging all the $\mathrm{M}_{i}$ in a sample, as

$\overline{\mathrm{M}}=\frac{\sum_{i=1}^{N_{g}} \mathrm{M}_{i}}{N_{g}}$ 
(a)

(c)
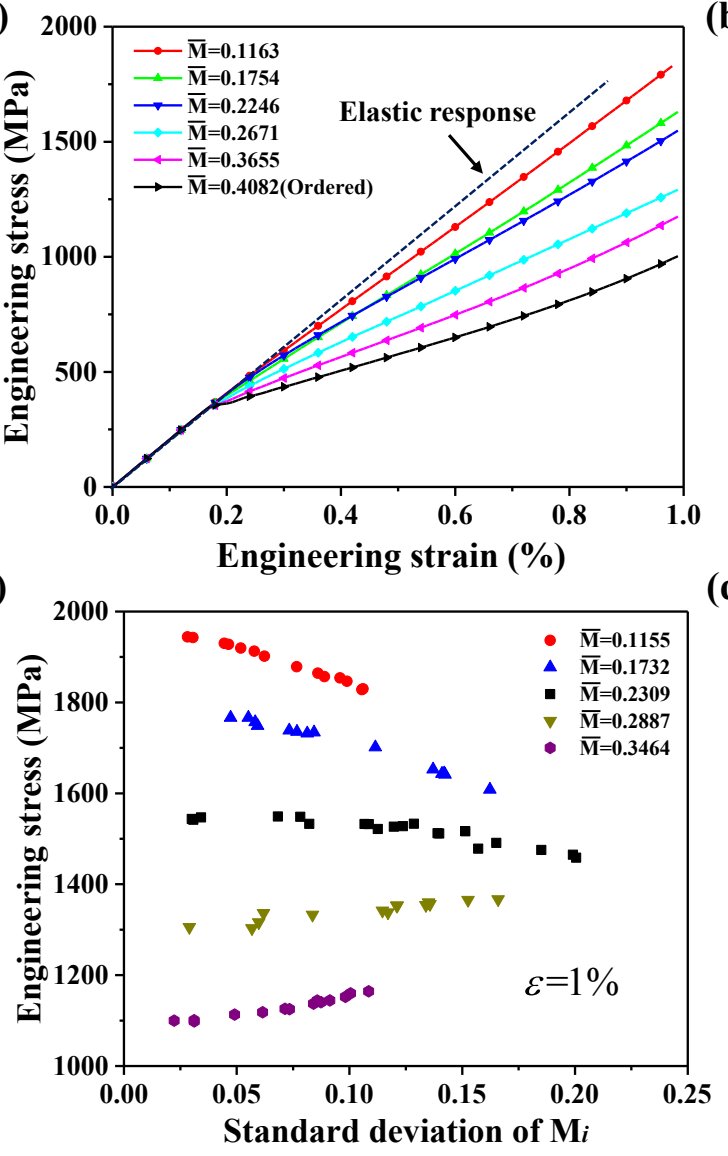

(b)

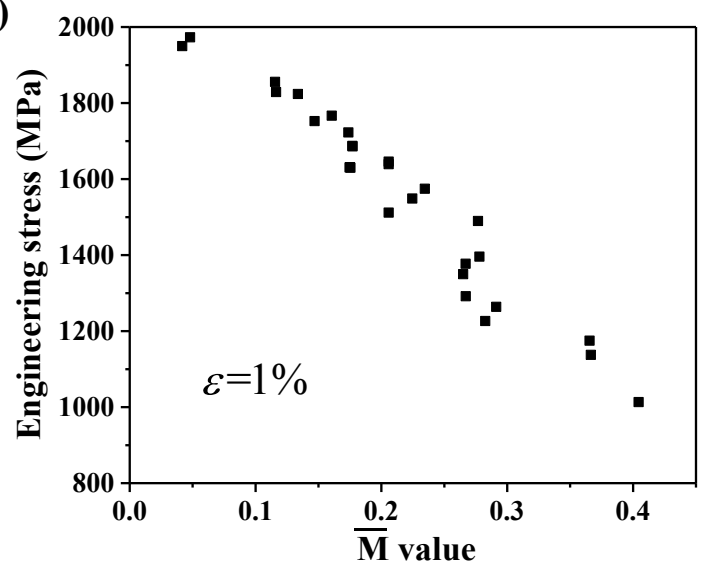

(d)

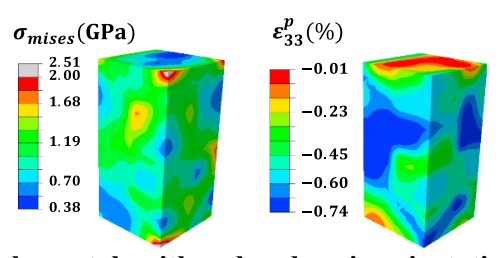

Polycrystals with ordered grain orientation

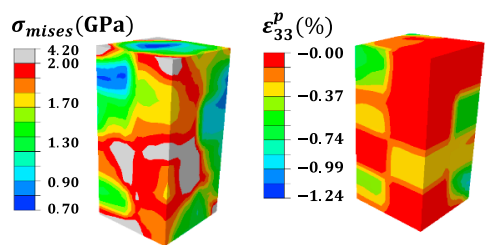

Polycrystals with disordered grain orientation

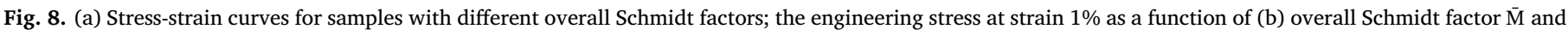

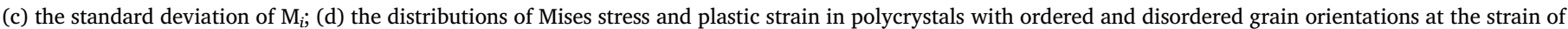
$1 \%$.

where $N_{g}$ is the number of grains in the sample. The standard deviation of these $\mathrm{M}_{i}$ is also evaluated and investigated.

Firstly, the engineering stress-strain curves for samples with different grain orientations which are evaluated with different $\bar{M}$ values are presented in Fig. 8(a). After the initial elastic deformation, yielding and hardening processes are also observed during the subsequent loading process. However, it can be seen from Fig. 8(a) that the sample with smaller overall Schmidt factor shows a more pronounced hardening phenomenon with a cost of decreasing ductility. That is, the higher strength means less plastic deformation and lower ductility. For simplicity, the ductility is evaluated by the stress level under the same deformation. Based on this idea, the engineering stress at strain $1 \%$ is shown in Fig. 8(b) to investigate the effect of grain orientation. Interestingly, the engineering stress decreases approximately linearly with the increase of overall Schmidt factor. It indicates that the grain orientation has a significant effect on the ductility of the polycrystals. The mobile dislocations in the grains with a larger Schmidt factor are more easily activated, resulting in more pronounced plasticity and exhibiting lower stress level. Moreover, since each grain in the polycrystalline sample has different local Schmidt factor, the standard deviation of $\mathrm{M}_{i}$ is further investigated to provide more insight into the effect of grain orientation. Through elaborate design of the grains in each sample, a series of polycrystalline nickel with the same overall Schmidt factor but different standard deviations are fabricated. Similarly, the engineering stresses at strain $1 \%$ for these polycrystalline nickel are shown in Fig. 8(c). For samples with different overall Schmidt factors, the stress distribution presents two opposite rules with the variation of standard deviation. For samples with smaller overall Schmidt factor, see $\bar{M}=0.1155$ and $\bar{M}=0.1732$ in Fig. 8(c), the stress decreases with the increase of standard deviation. It suggests that for polycrystals containing grains of different orientations, dislocations in grains with large local Schmidt factor are preferentially activated and dominate the plastic deformation. In more detail, for samples with $\bar{M}=0.1732$, when the standard deviation of local Schmidt factors becomes larger, some grains in the polycrystals have a larger Schmidt factor, thus the local resolved shear stress is larger in these grains which makes the dislocations easier to be activated and produce more plasticity. However, in Fig. 8(c), samples with larger overall Schmidt factor $(\bar{M}=0.2887)$ show the opposite characteristics. In the case, the number of grains with smaller local Schmidt factors increases as the standard deviation increases, as a result, internal dislocations are more difficult to be activated and the sample exhibits higher stress levels.

For the nano or micrograined metals, it is easy to achieve a remarkable strength but tend to be brittle [6], so the efforts to improve the material mechanical performance are usually concentrated on a better ductility. Accordingly, an issue arises that why the polycrystals with disordered grain orientation perform weaker ductility. To interpret the issue, the distributions of Mises stress and plastic strain in the samples are presented at the strain of $1 \%$, as shown in Fig. 8(d). It can be seen clearly that the distributions of stress and plastic strain are much more heterogeneous than those in sample with ordered grain orientation. The probability of deformation incompatibility between adjacent crystalline grains increases dramatically when the grain orientation is disorganized. The localized high stress makes the polycrystalline metals tend to form micro-cracks from grain boundaries. Instead, the continuity of slip systems in the samples with ordered grain orientation can avoid the stress concentration. Then the moderate deformation can continue so that the cracking process is suppressed. As a 
result, a better ductility can be achieved in the crystalline metals with ordered grain orientation. By further analyzing the dislocation density of the two models in Fig. 8(d), it is interesting that the overall dislocation density of sample with disordered grain orientation at the strain of $1 \%$ is $54.1 \mu \mathrm{m}^{-2}$, which is less than $78.5 \mu \mathrm{m}^{-2}$ in the sample of ordered grain orientation. It has been reported that the back stress hardening is caused by the pile-ups of dislocations [41]. In the disordered-orientation sample, it is more difficult to generate new dislocations due to the significant back stress. A few crystalline grains with high local dislocation density can lead to a weaker ductility. The results of DCP simulation indicate that it is the heterogeneity of dislocation distribution but not the total dislocation density which has more effects on the plastic behavior of polycrystalline materials.

\section{Discussion}

Polycrystalline metals with different grain sizes are expected to deform heterogeneously and only a part of crystalline grains undergo plastic deformation at the initial yielding [8]. It has also been reported that dislocations may no longer accumulate inside grains so that grain interiors are often dislocation free when the grain size is much smaller than $100 \mathrm{~nm}$ [4]. For NC metals with grain sizes range from $\sim 100 \mathrm{~nm}$ down to $\sim 10 \mathrm{~nm}$, a transition from dislocation glide dominated deformation to grain boundary mediated deformation is suggested by molecular simulations and experiments [42]. Consequently, the DCP model developed in this work is effective for polycrystals with grains at submicron scale by considering the dislocation glide deformation mechanism. It is worth mentioning that for polycrystalline materials in practical applications, the number of grains on the free surface is much less than that inside the sample such that the surface grains have very limited influence on the overall mechanical response of materials. In the simulation, the traction from free surface is not considered, so the simulation results are more suitable for bulk materials rather than finite-sized small samples.

The simulation results and analysis above highlight the effects of both grain size and grain orientation on the mechanical behavior of polycrystalline metals. The initial elastic rise in the stress-strain curves is due to that the driven force is not high enough to activate the dislocation sources and there is no plastic deformation at this stage. Then the yielding starts when the mobile dislocations are activated. The grain boundaries trap the dislocation mobility inside the grains, and thus introduce a pronounced back stress. The back stress promotes the material hardening and in turn decreases the ductility, which is the paradox of material strength and ductility.

The investigation of grain orientation provides new idea for the design of grain distribution in polycrystalline metals: The effect of grain orientation distribution on ductility shows opposite trends in different level of average Schmidt factor. The grains of large local Schmidt factor are termed as soft grains since the internal mobile dislocations can be more easily activated. Correspondingly, the grains of small local Schmidt factor are defined as hard grains. In general, samples with larger overall Schmidt factor exhibit a better ductility, i.e. a lower stress level at the same deformation, see Fig. 8(b). In addition, as shown in Fig. 8(c), polycrystals with $\bar{M}=0.2309$ can achieve an excellent balance of soft and hard grains. For samples with the overall Schmidt factor below this value, see $\bar{M}=0.1155$ and $\bar{M}=0.1732$ in Fig. 8(c), hard grains dominate the deformation, so as the standard deviation of $\mathrm{M}_{i}$ increases, the number of soft grains increases accordingly. As a result, the stress level will decrease. While for samples with the overall Schmidt factor above 0.2309 , the soft grains dominate. With the change of the standard deviation of $\mathrm{M}_{i}$, the stress level shows the opposite trend. The material design that combining the hard and soft phases is of significance to improve the mechanical properties of materials. This strategy has also been reported in many material designs to achieve an excellent strength-ductility synergy, e.g., a heterogeneous lamella structure which is characterized with soft micro-grained lamellae embedded in hard ultrafine-grained lamella matrix has been fabricated and exhibits unusual high strength and ductility [41], adding a soft elastic substrate under the metal film can greatly expand its ductility $[43,44]$, and the gradient structure in heterostructured materials with higher volume fraction of gradient domains exhibits higher synergetic strengthening [45].

\section{Conclusion}

In the present work, we studied the effect of grain boundary and grain orientation on the plasticity and ductility of polycrystalline metals, by using a dislocation-based crystal plasticity (DCP) model. The constitutive law of each individual crystalline grain is proposed based on the systematical DDD simulations of confined plasticity [26]. By taking the constitutive law into the finite element analysis at the grain level, the force balance and displacement continuity between adjacent crystalline grains can be achieved under the framework of continuum mechanics. The DCP model is verified and compared with the results of DDD simulation. It provides an effective way to take account of the SAS mechanism which is an important factor at submicron scale. Thereafter, a series of uniaxial compressive tests are performed to investigate the effects of grain boundary and grain orientation. The simulation results reveal that the increasing grain boundary density can increase the back stress and decrease ductility. The loss of ductility is due to the inhomogeneity of dislocation distribution and strain localization. In addition, the grain orientation effect is investigated by changing the Schmidt factor of grains. Polycrystals with larger overall Schmidt factor generally exhibit lower yield strength. The effect of grain orientation distribution on ductility shows opposite trends when the average Schmidt factor changes below or above $\sim 0.2309$.

\section{CRediT authorship contribution statement}

Jianqiao Hu: Conceptualization, Methodology, Software, Formal analysis, Writing - original draft. Zhuo Zhuang: Resources, Investigation, Writing - review \& editing. Fengxian Liu: Validation, Data curation. Xiaoming Liu: Conceptualization, Writing - review \& editing, Supervision, Funding acquisition. Zhanli Liu: Project administration, Investigation, Resources.

\section{Acknowledgments}

This work is supported by Science Challenge Project, No. TZ2018001 and the National Natural Science Foundation of China (Grant No. 11802310). The work of X.L. is supported by the National Natural Science Foundation of China (Grant No. 11772334, 11432014, 11672301), by Youth Innovation Promotion Association CAS (2018022), by the Strategic Priority Research Program of the Chinese Academy of Sciences, Grant No. XDB22040501.

\section{References}

[1] H. Zhang, Z. Jiang, J. Lian, Q. Jiang, Bulk nanostructured Cu with high strength and good ductility, Adv. Eng. Mater. 10 (2008) 41-45.

[2] C.C. Koch, D.G. Morris, K. Lu, A. Inoue, Ductility of nanostructured materials, MRS Bull. 24 (2013) 54-58.

[3] E. Ma, Recent progress in improving ductility of ultra-high strength nanostructured metals, Met. Mater. Int. 10 (2004) 527-531.

[4] Y. Zhao, Y. Zhu, E.J. Lavernia, Strategies for improving tensile ductility of bulk nanostructured materials, Adv. Eng. Mater. 8 (2010) 769-778.

[5] Y. Wang, M. Chen, F. Zhou, E. Ma, High tensile ductility in a nanostructured metal, Nature 419 (2002) 912-915.

[6] K. Lu, Making strong nanomaterials ductile with gradients, Science 345 (2014) 1455-1456.

[7] S. Lefebvre, B. Devincre, T. Hoc, Simulation of the Hall-Petch effect in ultra-fine grained copper, Mater. Sci. Eng., A 400-401 (2005) 150-153.

[8] G. Saada, Hall-Petch revisited, Mater. Sci. Eng., A 400-401 (2005) 146-149.

[9] E.O. Hall, The deformation and ageing of mild steel III discussion of results, Proc. Phys. Soc. B 64 (1951) 747-753.

[10] N.J. Petch, The cleavage strength of polycrystals, J. Iron Steel Inst. 173 (1953) 
$25-28$.

[11] V.T. Phan, T.D. Nguyen, Q.H. Bui, G. Dirras, Modelling of microstructural effects on the mechanical behavior of ultrafine-grained Nickel using crystal plasticity finite element model, Int. J. Eng. Sci. 94 (2015) 212-225.

[12] L. Li, P.M. Anderson, M.G. Lee, E. Bitzek, P. Derlet, H.V. Swygenhoven, The stress-strain response of nanocrystalline metals: a quantized crystal plasticity approach, Acta Mater. 57 (2009) 812-822.

[13] R. Yuan, I.J. Beyerlein, C. Zhou, Emergence of grain-size effects in nanocrystalline metals from statistical activation of discrete dislocation sources, Acta Mater. 90 (2015) 169-181.

[14] H. Zhang, X. Dong, D. Du, Q. Wang, A unified physically based crystal plasticity model for FCC metals over a wide range of temperatures and strain rates, Mater. Sci. Eng., A 564 (2013) 431-441.

[15] A. Arsenlis, D.M. Parks, R. Becker, V.V. Bulatov, On the evolution of crystallographic dislocation density in non-homogeneously deforming crystals, J. Mech. Phys. Solids 52 (2004) 1213-1246.

[16] B. von Blanckenhagen, E. Arzt, P. Gumbsch, Discrete dislocation simulation of plastic deformation in metal thin films, Acta Mater. 52 (2004) 773-784.

[17] C. Zhou, S.B. Biner, R. LeSar, Discrete dislocation dynamics simulations of plasticity at small scales, Acta Mater. 58 (2010) 1565-1577.

[18] G. Po, M.S. Mohamed, T. Crosby, C. Erel, A. El-Azab, N. Ghoniem, Recent progress in discrete dislocation dynamics and its applications to micro plasticity, Miner. Met. Mater. Soc. 66 (2014) 2108-2120.

[19] Y. Cui, P. Lin, Z. Liu, Z. Zhuang, Theoretical and numerical investigations of single arm dislocation source controlled plastic flow in FCC micropillars, Int. J. Plast. 55 (2014) 279-292.

[20] P. Lin, Z. Liu, Y. Cui, Z. Zhuang, A stochastic crystal plasticity model with sizedependent and intermittent strain bursts characteristics at micron scale, Int. J. Solids Struct. 69-70 (2015) 267-276.

[21] H. Yang, M. Huang, Z. Li, The influence of vacancies diffusion-induced dislocation climb on the creep and plasticity behaviors of nickel-based single crystal superalloy, Comput. Mater. Sci. 99 (2015) 348-360.

[22] S.S. Shishvan, L. Nicola, E. Van der Giessen, Bauschinger effect in unpassivated freestanding thin films, J. Appl. Phys. 107 (2010) 093529.

[23] Z. Li, C. Hou, M. Huang, C. Ouyang, Strengthening mechanism in micro-polycrystals with penetrable grain boundaries by discrete dislocation dynamics simulation and Hall-Petch effect, Comput. Mater. Sci. 46 (2009) 1124-1134.

[24] S.S. Quek, Z. Wu, Y.W. Zhang, D.J. Srolovitz, Polycrystal deformation in a discrete dislocation dynamics framework, Acta Mater. 75 (2014) 92-105.

[25] Y. Zhu, H. Wang, X. Zhu, Y. Xiang, A continuum model for dislocation dynamics incorporating Frank-Read sources and Hall-Petch relation in two dimensions, Int. J. Plast. 60 (2014) 19-39.

[26] Y. Cui, Z. Liu, Z. Zhuang, Theoretical and numerical investigations on confined plasticity in micropillars, J. Mech. Phys. Solids 76 (2015) 127-143.

[27] C. Zhou, I.J. Beyerlein, R. LeSar, Plastic deformation mechanisms of FCC single crystals at small scales, Acta Mater. 59 (2011) 7673-7682.

[28] I. Ryu, W. Cai, W.D. Nix, H. Gao, Stochastic behaviors in plastic deformation of face-centered cubic micropillars governed by surface nucleation and truncated source operation, Acta Mater. 95 (2015) 176-183.

[29] I. Groma, F.F. Csikor, M. Zaiser, Spatial correlations and higher-order gradient terms in a continuum description of dislocation dynamics, Acta Mater. 51 (2003) 1271-1281.

[30] P. Lin, Z. Liu, Z. Zhuang, Numerical study of the size-dependent deformation morphology in micropillar compressions by a dislocation-based crystal plasticity model, Int. J. Plast. 87 (2016) 32-47.

[31] C. Ouyang, Z. Li, M. Huang, C. Hou, Discrete dislocation analyses of circular nanoindentation and its size dependence in polycrystals, Acta Mater. 56 (2008) 2706-2717.

[32] Z. Liu, X. Liu, Z. Zhuang, X. You, A multi-scale computational model of crystal plasticity at submicron-to-nanometer scales, Int. J. Plast. 25 (2009) 1436-1455.

[33] Y. Cui, Z. Liu, Z. Zhuang, Quantitative investigations on dislocation based discretecontinuous model of crystal plasticity at submicron scale, Int. J. Plast. 69 (2015) $54-72$.

[34] J. Hu, Z. Liu, K. Chen, Z. Zhuang, Investigations of shock-induced deformation and dislocation mechanism by a multiscale discrete dislocation plasticity model, Comput. Mater. Sci. 131 (2017) 78-85.

[35] T.A. Parthasarathy, S.I. Rao, D.M. Dimiduk, M.D. Uchic, D.R. Trinkle, Contribution to size effect of yield strength from the stochastics of dislocation source lengths in finite samples, Scr. Mater. 56 (2007) 313-316.

[36] S.W. Lee, W.D. Nix, Size dependence of the yield strength of FCC and BCC metallic micropillars with diameters of a few micrometers, Phil. Mag. 92 (2012) 1238-1260.

[37] Y. Zhao, T. Topping, J.F. Bingert, J.J. Thornton, A.M. Dangelewicz, Ying Li, Wei Liu, Yuntian Zhu, Yizhang Zhou, E.J. Lavernia, High tensile ductility and strength in bulk nanostructured nickel, Adv. Mater. (2008) 3028-3033.

[38] A. Rinaldi, P. Peralta, C. Friesen, K. Sieradzki, Sample-size effects in the yield behavior of nanocrystalline nickel, Acta Mater. 56 (2008) 511-517.

[39] J.R. Greer, J.T.M. De Hosson, Plasticity in small-sized metallic systems: intrinsic versus extrinsic size effect, Prog. Mater. Sci. 56 (2011) 654-724.

[40] N. Hansen, Hall-Petch relation and boundary strengthening, Scr. Mater. 51 (2004) 801-806.

[41] X. Wu, M. Yang, F. Yuan, G. Wu, Y. Wei, X. Huang, Y. Zhu, Heterogeneous lamella structure unites ultrafine-grain strength with coarse-grain ductility, Proc. Natl. Acad. Sci. USA 112 (2015) 14501-14505.

[42] L. Capolungo, D.E. Spearot, M. Cherkaoui, D.L. McDowell, J. Qu, K.I. Jacob, Dislocation nucleation from bicrystal interfaces and grain boundary ledges: relationship to nanocrystalline deformation, J. Mech. Phys. Solids 55 (2007) 2300-2327.

[43] T. Li, Z. Suo, Deformability of thin metal films on elastomer substrates, Int. J. Solids Struct. 43 (2006) 2351-2363.

[44] N. Lu, Z. Suo, J.J. Vlassak, The effect of film thickness on the failure strain of polymer-supported metal films, Acta Mater. 58 (2010) 1679-1687.

[45] Y. Wang, C. Huang, M. Wang, Y. Li, Y. Zhu, Quantifying the synergetic strengthening in gradient material, Scr. Mater. 150 (2018) 22-25. 